\title{
Navigating Through Pandemic: The Use of Positioning Technologies
}

\author{
Anahid Basiri \\ (School of Geographical and Earth Sciences, University of Glasgow, Glasgow, UK) \\ (E-mails: ana.basiri@glasgow.ac.uk,journal@rin.org.uk)
}

The covid-19 pandemic and the enforced lockdown have disrupted many aspects of our lives, including our daily commutes and travels. For our community working on navigation, whether over land or sea or through the air, this is a particularly important time, as both our lives and our research topic have been affected. However, the value and importance of positioning, proximity and location have received more attention than usual. The core part of the mitigation plans of almost all countries is based on social distancing, and this certainly requires some sort of location (either distance and proximity or location). There have been a variety of approaches for managing the infection outbreaks, including mobile phone applications that should be able to identify and alert the potentially infected individuals who have been in contact with covid-19 patients.

Technologies that can help citizens to keep social distancing need to have certain qualities. Availability, reliability, privacy and accuracy are the most important quality aspects of the technologies to be implemented for a contact-tracing application. Due to the continuity requirement, the technology should be able to be used in all conditions and locations. Any contact-tracing application (app) needs to be a device-based technology (such as infrared or Bluetooth) rather than infrastructure or beacon based (such as wireless local area network (WLAN) or passive radio-frequency identification (RFID)). Although there are some contact-tracing apps that are based on QR codes or RFID technologies, their scalable use may need huge investment and new infrastructure to be deployed. For the covid-19 pandemic this time delay might compromise the purpose of the app, i.e., controlling the pandemic in the shortest possible time. Due to the privacy concerns associated with the contact-tracing data, proximity should not be calculated based on a device-free technology, such as CCTV cameras. Earlier research, including Basiri et al. (2017) and Mautz (2012), has reviewed more than 20 positioning and proximity sensing technologies from different quality aspects, including output data rate, accuracy, coverage or reach of the signals, cost to users or cost of new infrastructure deployment, power consumption, and privacy.

We assume the mobile phone cannot be modified or moderated in such a short period of time. If there is a possibility to deploy or install new beacons in a place, such as an office, then there are more options, such as ultrawideband (UWB). But considering the privacy requirement, power consumption that may limit the use, and the achievable accuracy of the technologies currently embedded in our mobile phones, we may end up with Bluetooth low energy (BLE) technology. 
BLE is a version of Bluetooth meant for low power applications, which allows some apps to operate in a continuous manner. A shorter operation range allows for proximitybased positioning, providing better performance regarding the estimated distance/ranging error (Simona-Lohan et al., 2015). The use of BLE for social distancing has gained a great deal of attention; however, there are some limitations that need to be discussed to appreciate the reliability and accuracy of the proximity sensing for such a sensitive application, i.e., social distancing at the time of covid-19:

(1) Sensitivity and high noise of BLE signals:

BLE signals are relatively sensitive and noisy. For example, the received signal strength index (RSSI) may decrease simply due to the way a phone is carried or the orientation of the device holder. This can introduce a challenge to an effective contact-tracing mobile app that should distinguish whether a person is within one- or two-metre proximity.

(2) Battery and sampling frequency balance:

While BLE is supposed to require low energy, the constant use may introduce a challenge for the user experience. If citizens must use the contact-tracing app whenever they are outside their homes, then power consumption needs to be considered as a key factor. Power consumption is highly correlated with the frequency of scanning. Leith and Farrell (2020) show that a widely-considered implementation designed by Google and Apple only scans every 3.5-5 min, which may not be ideal for some movement patterns that involve several 'epochs' of proximity accumulating the longer period of contact.

(3) High false-positive cases in detection of transmission, due to barriers such as walls, windows and partitions:

Barriers and partitions may not affect RSSI significantly enough to enable the app to detect them, while they can stop the transmission of the disease even though the distance between users is below the one- or two-metre proposed threshold.

BLE signal strength can vary due to several factors, including users (such as handgrip, speed and heading), environmental (such as furniture and indoor architecture) and device factors (such as device gain and orientation). Despite all these concerns, BLE has been implemented in many contact-tracing apps as there is no better technology currently embedded in our phones that can provide the required quality of service in a consistent way, seamlessly indoors and outdoors. For example, global navigation satellite systems (GNSS) are the most widely used positioning technologies, but as they provide the absolute location and also may only be available outdoors they have not been used in many of the contact-tracing apps. Due to privacy concerns associated with tracking and identifying the absolute location of users, in many countries several technologies cannot be used for social distancing purposes.

This certainly shows the importance of ubiquitous positioning and indoor navigation, which have been topics of research for many years. Only in this issue of the Journal several papers look at seamless positioning or the use of signals as opportunities to improve localisation. For example, Li et al. (2020) propose an improved fingerprinting algorithm that implements an access and reference point selection strategy to improve positioning accuracy. $\mathrm{Ng}$ et al. (2020) look at another aspect of seamless positioning which is multipath and reflection of signals. They provide a three-dimensional mapping aided GNSS positioning 
method for dense urban canyons, which can detect the signal reflection points based on a skyplot with the surrounding building boundaries. You et al. (2020) look at the challenges of signal-to-noise ratio and propose a wavelet basis design method based on frequency domain analysis to improve the denoising effect. And Peng et al. (2020) propose a method to address the challenge of positioning using GNSS in complex environments due to the correlation between ambiguity and the phase difference inter-system bias.

While these studies were not conducted directly and solely for the purpose of social distancing, they all look at different aspects of positioning and navigation in difficult environments, such as urban canyons or indoors. These will build a pathway for higher quality of services to society and in cities. A good example of the applied research in this issue is the paper by Elhajj and Ochieng (2020) that analyses for the first time the impact of new GPS signals on positioning accuracy for dynamic urban applications, taking bus operations as an example. In the positioning domain, there is a significant improvement in two-dimensional and three-dimensional accuracy from dual frequency code measurements over the single frequency measurements, of $39 \%$ and $48 \%$ respectively, enabling more bus operation services to be supported.

\section{REFERENCES}

Basiri, A., Lohan, E. S., Moore, T., Winstanley, A., Peltola, P., Hill, C., Amirian, P. and Figueiredo e Silva, P. (2017). Indoor location based services challenges, requirements and usability of current solutions. Computer Science Review, 1-128.

Elhajj, M. and Ochieng, W. (2020). Impact of new GPS signals on positioning accuracy for urban bus operations. Journal of Navigation, 73, 1284-1305.

Leith, D. and Farrell, S. (2020). GAEN Due Diligence: Verifying the Google/Apple Covid Exposure Notification API. CoronaDef21, Proceedings of NDSS'21 2021.

Li, C., Huang, H. and Liao, B. (2020). An improved fingerprint algorithm with access point selection and reference point selection strategies for indoor positioning. Journal of Navigation, 73, 1182-1201.

Mautz, R. (2012). Indoor Positioning Technologies. ETH Library, Zurich, Switzerland.

$\mathrm{Ng}$, H., Zhang, G. and Hsu, L. (2020). A computation effective range-based 3D mapping aided GNSS with NLOS correction method. Journal of Navigation, 73, 1202-1222.

Peng, Z., Gao, C. and Shang, R. (2020). Maximum ratio principle-based estimation of difference inter-system bias. Journal of Navigation, 73, 1372-1386.

Simona-Lohan, E., Talvitie, J., Figueiredo e Silva, P., Nurminen, H., Ali-Löytty, S. and Piché, R. (2015) Received Signal Strength Models for WLAN and BLE-Based Indoor Positioning in Multi-Floor Buildings. In: 2015 International Conference on Localization and GNSS (ICL-GNSS), Gothenberg, Sweden, pp. 1-6.

You, S., Wang, H., He, Y., Xu, Q. and Feng, L. (2020). Frequency domain design method of wavelet basis based on pulsar signal. Journal of Navigation, 73, 1223-1236. 